\title{
Decomposition of fuzzy ideal continuity via fuzzy idealization
}

\author{
Ahmed M. Zahran ${ }^{\star}$, S. A. Abd El-Baki ${ }^{\star \star}$, \\ Yaser M. Saber ${ }^{\star}$ \\ * Department of Mathematics, Faculty of Science, Al-Azhar University, Assiut 71524, Egypt \\ ** Department of Mathematics, Faculty of Science, Assiut University, Assiut 71524, Egypt
}

\begin{abstract}
Recently, El-Naschie has shown that the notion of fuzzy topology may be relevant to quantum paretical physics in connection with string theory and E-infinity space time theory. In this paper, we study the concepts of r-fuzzy semi-I-open, r-fuzzy pre-I-open, r-fuzzy $\alpha$-I-open and r-fuzzy $\beta$-I-open sets, which is properly placed between r-fuzzy openness and r-fuzzy $\alpha$-I-openness (r-fuzzy pre-I-openness) sets regardless the fuzzy ideal topological space in $\hat{S}$ ostak sense. Moreover, we give a decomposition of fuzzy continuity, fuzzy ideal continuity and fuzzy ideal $\alpha$-continuity, and obtain several characterization and some properties of these functions. Also, we investigate their relationship with other types of function.
\end{abstract}

Key words : r-fuzzy semi-I-open, r-fuzzy pre-I-open, r-fuzzy $\alpha$-I-open and r-fuzzy $\beta$-I-open sets, fuzzy ideal continuity and fuzzy ideal $\alpha$-continuity.

\section{Introduction and Preliminaries}

The concept of fuzzy topology was first defined in 1968 by Chang [1] and later redefined in a somewhat different way by Lowen [21] and by Hutton and Reilly [18]. According to Śstak's [27], in all these definitions, a fuzzy topology is a crisp subfamily of fuzzy sets and fuzziness in the concept of openness of a fuzzy set has not been considered, which seems to be a drawback in the process of fuzzification of the concept of topological spaces. Therefore Sostak's introduced a new definition of fuzzy topology in 1985 [28]. Later on, he developed the theory of fuzzy topological spaces in [29]. After that several authors $[2,3,5,19,20,23,25]$ have introduced the smooth definition and studied smooth fuzzy topological spaces being unaware of Sostak's works. In fuzzy topology, by introducing the notion of ideal, [27], and several other authors $[17,22]$ carried out such analysis.

The notion of continuity is an important concept in fuzzy topology and fuzzy topology in $\hat{S}$ ostak sense as well as in all branches of mathematics and quantum physics (see $[6,7,10,11,13,14])$. We must state that this subject has been researched by physicists $[7,1013]$ as well as by others. ElNaschie has shown that the notion of fuzzy topology in Sostak sense has very important applications in quantum particle physics especially in relation to both string theory and $\varepsilon^{(\infty)}$ theory $[8,9,12,15,16]$. In this paper, we give a decomposition of fuzzy continuity, fuzzy ideal continuity and fuzzy ideal $\alpha$-continuity, and we obtain several character-

Manuscript received Dec. 28. 2008; revised May. 16. 2009. izations of fuzzy $\alpha$-I-continuous functions. Moreover, we introduce the concept of fuzzy $\alpha$-I-open functions in fuzzy ideal topological spaces and obtain their properties

Throughout this paper, let $X$ be a nonempty set $I=[0,1]$ and $I_{0}=(0,1]$. For $\alpha \in I, \bar{\alpha}(x)=\alpha$ for all $x \in X$. The family of all fuzzy sets on $X$ denoted by $I^{X}$. For two fuzzy sets we write $\lambda q \mu$ to mean that $\lambda$ is quasi-coincident (q-coincident, for short) with $\mu$, i.e, there exists at least one point $x \in X$ such that $\lambda(x)+\mu(x)>1$. Negation of such a statement is denoted as $\lambda \bar{q} \mu$.

Definition 1.1 [27]. A mapping $\tau: I^{X} \rightarrow I$ is called a fuzzy topology on $\mathrm{X}$ if it satisfies the following conditions:

$$
\begin{aligned}
& \text { (O1) } \tau(\overline{0})=\tau(\overline{1})=1 \\
& \text { (O2) } \tau\left(\bigvee_{i \in \Gamma} \mu_{i}\right) \geq \bigwedge_{i \in \Gamma} \tau\left(\mu_{i}\right), \text { for }\left\{\mu_{i}\right\}_{i \in \Gamma} \in I^{X} \\
& \text { (O3) } \tau\left(\mu_{1} \wedge \mu_{2}\right) \geq \tau\left(\mu_{1}\right) \wedge \tau\left(\mu_{2}\right), \text { for } \mu_{1}, \mu_{2} \in I^{X}
\end{aligned}
$$

Definition 1.2 [26]. A mapping I : $I^{X} \rightarrow I$ is called fuzzy ideal on $\mathrm{X}$ iff:

$$
\begin{aligned}
& \left(I_{1}\right) \mathbf{I}(\underline{0})=1, \mathbf{I}(\underline{1})=0 . \\
& \left(I_{2}\right) \text { If } \lambda \leq \mu, \text { then } \mathbf{I}(\lambda) \geq \mathbf{I}(\mu), \text { for each } \lambda, \mu \in I^{X} . \\
& \left(I_{3}\right) \text { For each } \lambda, \mu \in I^{X}, \quad \mathbf{I}(\lambda \vee \mu) \geq \mathbf{I}(\lambda) \wedge \mathbf{I}(\mu) .
\end{aligned}
$$

The pair $(X, \tau, \mathbf{I})$ is called fuzzy ideal topological space (fits, for short)

Corollary 1.1. Let $(X, \tau, \mathcal{I})$ be a fits. The simplest fuzzy 
ideal on $X$ are $\mathcal{I}^{0}, \mathcal{I}^{1}: I^{X} \rightarrow I$ where

$\mathbf{I}^{0}(\lambda)=\left\{\begin{array}{l}1, \text { if } \lambda=\underline{0}, \\ 0, \text { otherwise. }\end{array} \quad \mathbf{I}^{1}(\lambda)=\left\{\begin{array}{l}0, \text { if } \lambda=\underline{1} \\ 1, \text { otherwise }\end{array}\right.\right.$

If $\mathbf{I}=\mathbf{I}^{0}$, for each $\mu \in I^{X}$ we have $\mu_{r}^{*}=C_{\tau}(\mu, r)$.

If $\mathbf{I}=\mathbf{I}^{1}$, for each $\mu \in \Theta^{\prime}$ we have $\mu_{r}^{*}=\underline{0}$, where, $\underline{1} \notin \Theta^{\prime}$ be a subset of $I^{X}$.

Definition 1.4 [4]. Let $(X, \tau, \mathbf{I})$ be a fits. Let $\mu, \lambda \in I^{X}$, the r-fuzzy open local function $\mu_{r}^{*}$ of $\mu$ is the union of all fuzzy points $x_{t}$ such that if $\rho \in Q\left(x_{t}, r\right)$ and $\mathbf{I}(\lambda) \geq r$ then there is at least one $y \in X$ for which $\rho(y)+\mu(y)-1>\lambda(y)$.

Theorem 1.1[3]. Let $(X, \tau)$ be a fts. Then for each $r \in I_{0}, \lambda \in I^{X}$ we define an operator $C_{\tau}: I^{X} \times I_{0} \rightarrow I^{X}$ as follows:

$$
C_{\tau}(\lambda, r)=\bigwedge\left\{\mu \in I^{X}: \lambda \leq \mu, \tau(\overline{1}-\mu) \geq r\right\}
$$

For $\lambda, \mu \in I^{X}$ and $r, s \in I_{0}$, the operator $C_{\tau}$ satisfies the following conditions:
(1) $C_{\tau}(\overline{0}, r)=\overline{0}$
(2) $\lambda \leq C_{\tau}(\lambda, r)$.
(3) $C_{\tau}(\lambda, r) \vee C_{\tau}(\mu, r)=C_{\tau}(\lambda \vee \mu, r)$.
(4) $C_{\tau}(\lambda, r) \leq C_{\tau}(\lambda, s)$ if $r \leq s$.
(5) $C_{\tau}\left(C_{\tau}(\lambda, r), r\right)=C_{\tau}(\lambda, r)$.

Theorem 1.2[24]. Let $(X, \tau)$ be a fts. Then for each $r \in I_{0}, \lambda \in I^{X}$ we define an operator $I_{\tau}: I^{X} \times I_{0} \rightarrow I^{X}$ as follows:

$$
I_{\tau}(\lambda, r)=\bigvee\left\{\mu \in I^{X}: \lambda \geq \mu, \tau(\mu) \geq r\right\} .
$$

For $\lambda, \mu \in I^{X}$ and $r, s \in I_{0}$, the operator $I_{\tau}$ satisfies the following conditions:
(1) $I_{\tau}(\overline{1}-\lambda, r)=\overline{1}-C_{\tau}(\lambda, r)$
(2) $I_{\tau}(\overline{1}, r)=\overline{1}$.
(3) $\lambda \geq I_{\tau}(\lambda, r)$.
(4) $I_{\tau}(\lambda, r) \wedge I_{\tau}(\mu, r)=I_{\tau}(\lambda \wedge \mu, r)$.
(5) $I_{\tau}(\lambda, r) \leq I_{\tau}(\lambda, s)$ if $r \geq s$.
(6) $I_{\tau}\left(I_{\tau}(\lambda, r), r\right)=I_{\tau}(\lambda, r)$.

Theorem 1.3[4]. Let $(X, \tau)$ be a fts and $\mathbf{I}_{1}, \mathbf{I}_{2}$ be two fuzzy ideals of $\mathrm{X}$. Then for each $r \in I_{0}$ and $\mu, \eta, \rho \in I^{X}$.

(1) $\mu \leq \eta$, then $\mu_{r}^{*} \leq \eta_{r}^{*}$.

(2) $\mathbf{I}_{1} \leq \mathbf{I}_{2}, \Rightarrow \mu_{r}^{*}\left(\mathbf{I}_{1}, \tau\right) \leq \eta_{r}^{*}\left(\mathbf{I}_{2}, \tau\right)$.

(3) $\mu_{r}^{*}=C_{\tau}\left(\mu_{r}^{*}, r\right) \leq C_{\tau}(\mu, r)$.

(4) $\left(\mu_{r}^{*}\right)^{*} \leq \mu_{r}^{*}$.

(5) $\left(\mu_{r}^{*} \vee \eta_{r}^{*}\right)=(\mu \vee \eta)_{r}^{*}$.

(6) If $\mathbf{I}(\rho) \geq r$ then $(\mu \vee \rho)_{r}^{*}=\mu_{r}^{*} \vee \rho_{r}^{*}=\mu_{r}^{*}$.

(7) If $\tau(\rho) \geq r$, then $\left(\rho \wedge \mu_{r}^{*}\right) \leq(\rho \wedge \mu)_{r}^{*}$.

(8) $\left(\mu_{r}^{*} \wedge \eta_{r}^{*}\right) \geq(\mu \wedge \eta)_{r}^{*}$.
Theorem 1.4[4]. Let $(X, \tau, \mathbf{I})$ be a fits. Then for each $r \in I_{0}, \mu \in I^{X}$ we define $C^{*}: I^{X} \times I_{0} \rightarrow I^{X}$ as follows:

$$
C l^{*}(\mu, r)=\mu \vee \mu_{r}^{*}
$$

For $\mu, \eta \in I^{X}$, the $C l^{*}$ satisfies the following conditions:
(1) If $\mu \leq \eta$, then $C l^{*}(\mu, r) \leq C l^{*}(\eta, r)$.
(2) $C l^{*}\left(C l^{*}(\mu, r), r\right)=C l^{*}(\mu, r)$.
(3) $C l^{*}(\mu \vee \eta, r)=C l^{*}(\mu, r) \vee C l^{*}(\eta, r)$.
(4) $C l^{*}(\mu \wedge \eta, r) \leq C l^{*}(\mu, r) \wedge C l^{*}(\eta, r)$.

Definition 1.5 [24]. Let $(X, \tau)$ be a fts. For $\lambda \in I^{X}$ and $r \in I_{0}$.

(1) $\lambda$ is called r-fuzzy semiopen (r-FSO, for short) iff $\lambda \leq C_{\tau}\left(I_{\tau}(\lambda, r), r\right)$.

(2) $\lambda$ is called r-fuzzy semiclosed (r-FSC, for short) iff $\overline{1}-\lambda$ is r-fuzzy semiopen set of $X$.

(3) $\lambda$ is called r-fuzzy $\beta$-closed ( $\mathbf{r}-\mathbf{F} \beta \mathbf{C}$, for short) iff $\lambda \leq C_{\tau}\left(I_{\tau}\left(C_{\tau}(\lambda, r), r\right), r\right)$.

Definition 1.6[4]. Let $(X, \tau, I)$ be a fuzzy ideal topological space. For each $\mu \in I^{X}$ and $r \in I_{0}$.

(1) $\mu$ is called r-fuzzy ideal open (r-FIO, for short) iff $\mu \leq I_{\tau}\left(\mu_{r}^{*}, r\right)$.

(2) $\mu$ is called r-fuzzy ideal closed (r-FIC, for short) iff $\overline{1}-\mu$ is r-FIO.

Lemma 1.1[4]. Let $(X, \tau, \mathbf{I})$ be a fits.

(1) Any union of r-FIO sets is r-FIO.

(2) Any intersection of r-FIC sets is r-FIC

Definition 1.7 [27]. Let $(X, \tau)$ and $(X, \eta)$ be fts's. Let $f: X \rightarrow Y$ be a mapping.

(1) $f$ is called fuzzy continuous iff $\eta(\mu) \leq \tau\left(f^{-1}(\mu)\right)$ for each $\mu \in I^{X}$.

(2) $f$ is called fuzzy open iff $\tau(\mu) \leq \eta(f(\mu))$ for each $\mu \in I^{X}$.

(3) $f$ is called fuzzy closed iff $\tau(\overline{1}-\mu) \leq \eta(f(\overline{1}-\mu))$ for each $\mu \in I^{X}$.

\section{2. r-fuzzy semi-I-open and r-fuzzy $\alpha$-I-open sets}

Definition 2.1. Let $(X, \tau, I)$ be a fuzzy ideal topological space, for each $\mu \in I^{X}$ and $r \in I_{0}$.

(1) $\mu$ is called r-fuzzy semi-I-open (r-FSIO, for short) iff $\mu \leq C l^{*}\left(I_{\tau}(\mu, r), r\right)$.

(2) $\mu$ is called r-fuzzy pre-ideal open (r-FPIO, for short) iff $\mu \leq I_{\tau}\left(C l^{*}(\mu, r), r\right)$. The complement of a $\mathrm{r}-$ fuzzy pre-ideal open set is said to be r-fuzzy pre-ideal closed (r-FPIC, for short.)

(3) $\mu$ is called r-fuzzy $\alpha$-ideal open (r-F $\alpha \mathbf{I O}$, for short) iff $\mu \leq I_{\tau}\left(C l^{*}\left(I_{\tau}(\mu, r), r\right)\right.$, ). The complement of a $\mathrm{r}$ fuzzy $\alpha$-ideal open set is said to be r-fuzzy $\alpha$-ideal closed (r-F $\alpha \mathbf{I C}$, for short.) 
(4) $\mu$ is called r-fuzzy $\beta$-ideal open (r-F $\beta \mathbf{I C}$, for short) iff $\mu \leq C_{\tau}\left(I_{\tau}\left(C l^{*}(\mu, r), r\right), r\right)$. The complement of a rfuzzy $\beta$-ideal open set is said to be r-fuzzy $\beta$-ideal closed (r-F $\beta \mathbf{I C}$, for short.)

Theorem 2.1. Let $(X, \tau, \mathbf{I})$ be a fits.

(1) Every r-fuzzy open set is r-F $\alpha \mathbf{I O}$

(2) Every $\mathbf{r - F} \alpha \mathbf{I O}$ set is r-FSIO.

(3) Every r-FSIO set is r-F $\beta$ IO.

(4) Every r-F $\alpha$ IO set is r-FPIO.

(5) Every r-FPIO set is r-F $\beta$ IO.

(6) Every r-FPIO set is r-FPO.

(7) Every r-fuzzy open set is r-FSIO.

(8) Every r-FSIO set is r-FSO.

(9) Every r-fuzzy open set is r-FPIO .

(10) Every r-FIO set is r-FPIO.

(11) Every r-F $\beta \mathbf{I O}$ set is $\mathrm{r}-\mathbf{F} \beta \mathbf{O}$.

Proof. (1) Let $\mu$ be r-fuzzy open set. Then

$$
\begin{aligned}
\mu & =I_{\tau}(\mu, r) \\
& \leq I_{\tau}(\mu, r) \vee\left(I_{\tau}(\mu, r)\right)^{*} \\
& =C l^{*}\left(I_{\tau}(\mu, r), r\right) .
\end{aligned}
$$

Therefore, $\mu=I_{\tau}(\mu, r) \leq I_{\tau}\left(C l^{*}\left(I_{\tau}(\mu, r), r\right), r\right)$. Implies that $\mu$ is $\mathbf{r}-\mathbf{F} \alpha \mathbf{I O}$.

(2) Let $\mu$ be r-F $\alpha$ IO. Then by Theorem 1.4(1),

$$
\mu \leq I_{\tau}\left(C l^{*}\left(I_{\tau}(\mu, r), r\right) \leq C l^{*}\left(I_{\tau}(\mu, r), r\right) .\right.
$$

(3) Let $\mu$ be r-FSIO. Then

$$
\begin{aligned}
\mu & \leq C l^{*}\left(I_{\tau}(\mu, r), r\right) \\
& \leq I_{\tau}(\mu, r) \vee\left(I_{\tau}(\mu, r)\right)^{*} \\
& \leq \mu \vee \mu_{r}^{*} \\
& \leq C l^{*}\left(I_{\tau}\left(\mu \vee \mu_{r}^{*}, r\right)\right. \\
& \leq C l^{*}\left(I_{\tau}\left(C l^{*}(\mu, r), r\right), r\right) \\
& \leq C_{\tau}\left(I_{\tau}\left(C l^{*}(\mu, r), r\right), r\right) .
\end{aligned}
$$

(4) Let $\mu$ be r-F $\alpha \mathbf{I O}$ set. Then

$$
\begin{aligned}
\mu & \leq I_{\tau}\left(C l^{*}\left(I_{\tau}(\mu, r), r\right)\right) \\
& =I_{\tau}\left(I_{\tau}(\mu, r) \vee\left(I_{\tau}(\mu, r)\right)^{*}\right) \\
& \leq I_{\tau}\left(\mu \vee \mu_{r}^{*}\right) \\
& =I_{\tau}\left(C l^{*}(\mu, r), r\right) .
\end{aligned}
$$

(5-10) This proof is obvious.

Remark 2.1. By Theorem 2.1, we obtain the diagram for a r-fuzzy ideal topological space:

$$
\begin{aligned}
& \text { r-fuzzy open } \Rightarrow r-\text { F } \alpha \text { IO } \Rightarrow r-\text { FSIO } \Rightarrow \text { r-FSO } \\
& \Downarrow \quad \Downarrow \quad \Downarrow \\
& r-\text { FIO } \Rightarrow r-\text { FPIO } \Rightarrow r-\text { F } \beta \text { IO } \Rightarrow \text { r-F } \beta \text { O }
\end{aligned}
$$

Remark 2.2. r-FPIO and r-FSIO are independent notions as show by the following Examples 2.1. and 2.2.

Example 2.1. Define two fuzzy topologies and fuzzy ideal $\tau, \mathbf{I}: I^{X} \rightarrow I$ as follows:

$$
\tau(\lambda)=\left\{\begin{array}{l}
1, \text { if } \lambda=\overline{1}, \overline{0} \\
\frac{1}{2}, \text { if } \lambda=\overline{0.4} \\
0, \text { Otherwise }
\end{array}\right.
$$

If we take $\mathbf{I}=\mathbf{I}^{0}$ for all $r \in I_{0}$, and let $\mu=\overline{0.3}$, then $\mu$ is $\frac{1}{2}$-FPIO, but $\mu$ is not $\frac{1}{2}$-FSIO.

Example 2.2. Let $X=\{a, b, c\}$ be a set and $a_{t} \in P_{t}(X)$. Define $\mu_{1} \mu_{2} \in I^{X}$ as follows:

$$
\begin{aligned}
& \mu_{1}(a)=0.2, \mu_{1}(b)=0.3, \mu_{1}(c)=0.7 \\
& \mu_{2}(a)=0.1, \mu_{2}(b)=0.2, \mu_{2}(c)=0.2 .
\end{aligned}
$$

We define $\tau, \mathbf{I}: I^{X} \rightarrow I$ as follows:

$$
\tau(\lambda)=\left\{\begin{array}{l}
1, \text { if } \quad \lambda=\overline{1}, \overline{0} \\
\frac{1}{2}, \text { if } \lambda=\mu_{2} \\
0, \quad \text { otherwise }
\end{array}\right.
$$

If we take $\mathbf{I}=\mathbf{I}^{0}$ for all $r \in I_{0}$, then $\mu_{1}$ is $\frac{1}{2}$-FSIO, but $\mu$ is not $\frac{1}{2}$-FPIO.

Remark 2.3. r-FIO and r-FSIO are independent notions as show by the following Examples 2.1. and 2.3.

Example 2.3. Define two fuzzy topologies and fuzzy ideal $\tau, \mathbf{I}: I^{X} \rightarrow I$ as follows:

$$
\tau(\lambda)=\left\{\begin{array}{l}
1, \text { if } \quad \lambda=\overline{1}, \overline{0} \\
\frac{1}{2}, \text { if } \quad \lambda=\overline{0.4} \\
\frac{2}{3}, \text { if } \lambda=\overline{0.6} \\
0, \quad \text { otherwise }
\end{array}\right.
$$

If we take $\mathbf{I}=\mathbf{I}^{0}$ for all $r \in I_{0}$, and let $\mu=\overline{0.5}$, then $\mu$ is $\frac{1}{2}$-FIO, but $\mu$ is not $\frac{1}{2}$-FSIO.

On the other hand, In Example 2.1. If we take $\mathbf{I}=\mathbf{I}^{0}$ for all $r \in I_{0}$, and let $\mu=\overline{0.6}$, then $\mu$ is $\frac{1}{2}$-FSIO but $\mu$ is not $\frac{1}{2}$-FIO.

Remark 2.4. r-fuzzy open set and r-FIO are independent notions as show by the following Example 2.1. and 2.4 .

Example 2.4. Define two fuzzy topologies and fuzzy ideal $\tau, \mathbf{I}: I^{X} \rightarrow I$ as follows:

$$
\tau(\lambda)=\left\{\begin{array}{lc}
1, \text { if } & \lambda=\overline{1}, \overline{0} \\
\frac{1}{2}, \text { if } & \lambda=\overline{0.4} \\
\frac{1}{3}, \text { if } & \lambda=\overline{0.3} \\
0, & \text { otherwise }
\end{array}\right.
$$


If we take $\mathbf{I}=\mathbf{I}^{1}$, for all $r \in I_{0}$, and let $\mu=\overline{0.3}$, then $\tau(\mu) \geq \frac{1}{3}$, but $\mu$ is not $\frac{1}{3}$-FIO.

On the other hand, In Example 2.1. If we take $\mathbf{I}=\mathbf{I}^{0}$ for all $r \in I_{0}$, and let $\mu=\overline{0.3}$, then $\mu$ is $\frac{1}{2}$-FIO, but $\tau(\mu)<\frac{1}{2}$.

Corollary 2.1. Let $(X, \tau, \mathbf{I})$ be a fits. For each $\mu \in I^{X}$.

(1) If $\mathbf{I}=\mathbf{I}^{0}$ for all $r \in I_{0}$, then,

(i) r-FIO, r-FPIO and r-FPO are equivalent,

(ii) $\mu$ r-FSIO if and only if r-FSO,

(iii) $\mu \mathrm{r}-\mathbf{F} \beta \mathbf{I O}$ if and only if $\mu$ is $\mathrm{r}-\mathbf{F} \beta \mathbf{O}$.

(2) If $\mathbf{I}=\mathbf{I}^{1}$ for all $r \in I_{0}$, then, $\mu$ is $\mathbf{r - F} \beta \mathbf{I O}$ if and only if $\mu$ is r-FSO.

Proof. (1) If $I=\mathbf{I}^{0}$ for all $r \in I_{0}$, then, $\mu_{r}^{*}=C_{\tau}(\mu, r)$ for any $\mu \in I^{X}$ and hence $C l^{*}(\mu, r)=\mu \vee \mu_{r}^{*}=C_{\tau}(\mu, r)$. Therefore, we have $\mu_{r}^{*}=C_{\tau}(\mu, r)=C l^{*}(\mu, r)$. Thus, (i), (ii), and (iii) follow immediately.

(2) If $\mathbf{I}=\mathbf{I}^{1}$ for all $r \in I_{0}$, then, $\mu_{r}^{*}=\overline{0}$. Therefore, we have $C_{\tau}\left(I_{\tau}\left(C l^{*}(\mu, r), r\right), r\right)=C_{\tau}\left(I_{\tau}\left(\mu_{r}^{*} \vee \mu, r\right), r\right)=$ $C_{\tau}\left(I_{\tau}(\mu, r), r\right)$. Thus, $\mathrm{r}-\mathbf{F} \beta \mathbf{I O}$ and $\mathbf{r}-\mathbf{F S O}$ are equivalent.

Definition 2.2. Let $(X, \tau, \mathbf{I})$ be a fits. For $\mu, \lambda \in I^{X}$ and $r \in I_{0}$.

(1) $\mu$ is called r-fuzzy t-I-set if

$$
I_{\tau}\left(C l^{*}(\mu, r), r\right)=I_{\tau}(\mu, r) .
$$

(2) $\mu$ is called r-fuzzy B-I-set if $\mu=\nu \wedge \lambda$, where $\tau(\nu) \geq r$ and $\lambda$ is r-fuzzy t-I-set of $\mathrm{X}$.

(3) $\mu$ is called r-fuzzy $*$-dense-in-itself if $\mu \leq \mu_{r}^{*}$.

Corollary 2.2. Let $(X, \tau, \mathbf{I})$ be a fits and $\lambda \in I^{X}$, the following properties are holds

(1) Every r-fuzzy t-I-set is r-fuzzy B-I-set.

(2) Every r-fuzzy $*$-dense-in-itself set is r-fuzzy t-I-set.

Proof. (1) Let $\mu$ is r-fuzzy t-I-set. Since $\mu=\overline{1} \wedge \mu$ then $\mu$ is a r-fuzzy B-I-set.

(2) Let $\mu$ is r-fuzzy $*$-dense-in-itself set. Then $I_{\tau}\left(C l^{*}(\mu, r), r\right)=I_{\tau}\left(\left(\mu_{r}^{*} \vee \mu, r\right)=I_{\tau}(\mu, r)\right.$.

Lemma 2.1. Let $(X, \tau, \mathbf{I})$ be a fits, for $\mu \in I^{X}$. The following statements are equivalent.

(1) $\mu$ is r-F $\alpha \mathbf{I O}$.

(2) $\mu$ r-FSIO and r-FPIO.

Proof. Necessity. This is obvious. have

Sufficiency. Let $\mu$ be r-FSIO and r-FPIO. Then, we

$$
\begin{aligned}
\mu & \leq I_{\tau}\left(C l^{*}(\mu, r), r\right) \\
& \leq I_{\tau}\left(C l^{*}\left(C l^{*}\left(I_{\tau}(\mu, r), r\right), r\right), r\right) \\
& =I_{\tau}\left(C l^{*}\left(I_{\tau}(\mu, r), r\right), r\right)
\end{aligned}
$$

This show that $\mu$ is $\mathbf{r}-\mathbf{F} \alpha \mathbf{I O}$.
Lemma 2.2. Let $(X, \tau, \mathbf{I})$ be a fits, for $\mu \in I^{X}$, the following statements are equivalent.

(1) $\mu$ is r-FIO.

(2) $\mu$ are r-FIPO and r-fuzzy $*$-dense-in-itself.

Proof. $(1 \Rightarrow 2)$ : by Theorem 2.1 , every r-FIO is r-FPIO. On the other hand, $\mu \leq I_{\tau}\left(\mu_{r}^{*}, r\right) \leq \mu_{r}^{*}$, which show that $\mu$ is r-fuzzy $*$-dense-in-itself.

$(2 \Rightarrow 1)$ : by the hypothesis, $\mu \leq I_{\tau}\left(C l^{*}(\mu, r), r\right) \leq$ $I_{\tau}\left(\mu \vee \mu_{r}^{*}, r\right)=I_{\tau}\left(\mu_{r}^{*}, r\right)$, then, $\mu$ is r-FIO.

Lemma 2.3. Let $(X, \tau, \mathbf{I})$ be a fits, for $\mu \in I^{X}$, the following statements are equivalent.

(1) $\tau(\mu) \geq r$.

(2) $\mu$ are r-FIPO and r-fuzzy B-I-set.

Proof. Let $\tau(\mu) \geq r$. Then $\mu \wedge \overline{1}$ follows that $\mu$ is a r-fuzzy B-I-set. $\mu$ is also r-FPIO by Theorem 2.1(9). Conversely, Let $\mu$ be both r-fuzzy B-I-set and r-FPIO. Then, $\mu \leq I_{\tau}\left(C l^{*}(\mu, r), r\right)$ and $\mu=\lambda \wedge \omega$ where $\tau(\lambda) \geq r$ and $\omega$ is r-fuzzy t-I-set. Therefore,

$$
\begin{aligned}
\lambda \wedge \omega & \leq I_{\tau}\left(C l^{*}(\lambda \wedge \omega, r), r\right) \\
& \leq I_{\tau}\left(C l^{*}(\lambda, r), r\right) \wedge I_{\tau}\left(C l^{*}(\omega, r), r\right) \\
& =I_{\tau}\left(C l^{*}(\lambda, r), r\right) \wedge I_{\tau}(\omega, r) .
\end{aligned}
$$

Hence,

$$
\begin{aligned}
\lambda \wedge \omega & \leq(\lambda \wedge \omega) \wedge \lambda \\
& =I_{\tau}\left(C l^{*}(\lambda, r), r\right) \wedge I_{\tau}(\omega, r) \wedge \lambda \\
& =\lambda \wedge I_{\tau}(\omega, r) .
\end{aligned}
$$

Thus, we obtain $\lambda \wedge \omega=\lambda \wedge I_{\tau}(\nu, r)$, implies $\tau(\mu) \geq r$.

Lemma 2.4. Let $(X, \tau, \mathbf{I})$ be a fuzzy ideal topological space and $\mu, \omega \in I^{X}$. If $\tau(\omega) \geq r$, then $\omega \wedge C l^{*}(\mu, r) \leq$ $C l^{*}(\mu \wedge \omega, r)$.

Proof. Let $\tau(\omega) \geq r$, by Theorem 1.3, then we have $\left(\omega \wedge \mu_{r}^{*}\right) \leq(\omega \wedge \mu)_{r}^{*}$ for any $\mu \in I^{X}$. Thus, we have

$$
\begin{aligned}
\omega \wedge C l^{*}(\mu, r) & =\omega \wedge\left(\mu \vee \mu_{r}^{*}\right) \\
& =(\omega \wedge \mu) \vee\left(\omega \wedge \mu_{r}^{*}\right) \\
& \leq(\omega \wedge \mu) \vee(\omega \wedge \mu)_{r}^{*} \\
& =C l^{*}(\omega \wedge \mu, r) .
\end{aligned}
$$

Theorem 2.2. Let $(X, \tau, \mathbf{I})$ be a fits and $\mu, \omega \in I^{X}$. Then the following properties hold: FSIO

(1) If $\mu$ is r-FSIO and $\omega$ is $\mathbf{r}-\mathbf{F} \alpha \mathbf{I O}$, then $\mu \wedge \omega$ is r-

(2) If $\mu$ is r-FPIO and $\omega$ is $\mathbf{r - F} \alpha \mathbf{I O}$, then $\mu \wedge \omega$ is rFPIO.

(3) If $\tau(\mu) \geq r$ and $\omega$ is r-FPIO, then $\mu \wedge \omega$ is r-FPIO.

(4) If $\tau(\mu) \geq r$ and $\omega$ is r-FSIO, then $\mu \wedge \omega$ is r-FSIO 
Proof. (1) Let $\mu$ be r-FSIO and $\omega$ be r-F $\alpha$ IO. By using Lemma 2.4, we have

$$
\begin{aligned}
\mu \wedge \omega & \leq C l^{*}\left(I_{\tau}(\mu, r), r\right) \wedge I_{\tau}\left(C l^{*}\left(I_{\tau}(\omega, r), r\right), r\right) \\
& \leq C l^{*}\left(I_{\tau}(\mu, r) \wedge C l^{*}\left(I_{\tau}(\omega, r), r\right), r\right) \\
& \leq C l^{*}\left(C l^{*}\left(I_{\tau}(\mu, r), r\right) \wedge I_{\tau}(\omega, r), r\right) \\
& \leq C l^{*}\left(I_{\tau}(\mu, r), r\right) .
\end{aligned}
$$

This show that $\mu \wedge \omega$ is r-FSIO.

(2-4) Similarly.

Corollary 2.3. Let $(X, \tau, \mathbf{I})$ be a fits and $\mu, \omega \in I^{X}$. Then the following properties hold:

(1) If $\mu$ is r-FSIO and $\tau(\omega) \geq r$, then $\mu \wedge \omega$ is r-FSIO

(2) If $\mu$ is r-FPIO and $\tau(\omega) \geq r$, then $\mu \wedge \omega$ is r-FPIO.

Theorem 2.3. Let $(X, \tau, \mathbf{I})$ be a fits and $\mu, \omega \in I^{X}$. Then the following properties hold:

(1) If $\mu$ and $\omega$ are $r-\mathbf{F} \alpha \mathbf{I O}$, then $\mu \wedge \omega$ is $\mathbf{r}-\mathbf{F} \alpha \mathbf{I O}$.

(2) If $\mu_{\gamma}$ is $\mathbf{r}-\mathbf{F} \alpha \mathbf{I O}$ for $\gamma \in \sigma$, then $\bigvee_{\gamma \in \sigma} \mu_{\gamma}$ is $\mathbf{r}-\mathbf{F} \alpha \mathbf{I O}$.

(3) If $\mu_{\gamma}$ is r-FPIO for $\gamma \in \sigma$, then $\bigvee_{\gamma \in \sigma} \mu_{\gamma}$ is r-FPIO.

Proof. (1) Let $\mu$ and $\omega$, be $\mathbf{r}-\mathbf{F} \alpha \mathbf{I O}$, by Lemma $2.1, \mu$ is r-FSIO and r-FPIO and by Theorem 2.2(1,2), $\mu \wedge \omega$ is r-FSIO and r-FPIO. Therefore, by Lemma $2.1, \mu \wedge \omega$ is r-F $\alpha \mathbf{I O}$.

(2) Let $\mu_{\gamma}$ be a class of $\mathbf{r}-\mathbf{F} \alpha \mathbf{I O}$. Then for any $\gamma \in \sigma$,

$$
\begin{aligned}
\mu_{\gamma} & \leq I_{\tau}\left(C l^{*}\left(I_{\tau}\left(\mu_{\gamma}, r\right), r\right), r\right) \\
& \leq I_{\tau}\left(C l^{*}\left(I_{\tau}\left(\bigvee_{\gamma \in \sigma} \mu_{\gamma}, r\right), r\right), r\right) .
\end{aligned}
$$

Hence $\bigvee_{\gamma \in \sigma} \mu_{\gamma} \leq I_{\tau}\left(C l^{*}\left(I_{\tau}\left(\bigvee_{\gamma \in \sigma} \mu_{\gamma}, r\right), r\right), r\right)$. This show that $\bigvee_{\gamma \in \sigma} \mu_{\gamma}$ is $\mathbf{r}-\mathbf{F} \alpha \mathbf{I O}$.

(3) Similarly.

Theorem 2.4. Let $(X, \tau, \mathbf{I})$ be a fits, if $\mu$ is r-FPIC then $C l^{*}\left(I_{\tau}(\mu, r), r\right) \leq \mu$, for each $\mu \in I^{X}$.

Proof. Let $\mu$ be r-FPIC. Then $\overline{1}-\mu$ is r-FPIO. Hence

$$
\begin{aligned}
\overline{1}-\mu & \leq I_{\tau}\left(C l^{*}(\overline{1}-\mu, r), r\right) \\
& \leq I_{\tau}\left(C_{\tau}(\overline{1}-\mu, r), r\right) \\
& =\overline{1}-C_{\tau}\left(I_{\tau}(\mu, r), r\right) \\
& \leq \overline{1}-C l^{*}\left(I_{\tau}(\mu, r), r\right) .
\end{aligned}
$$

Therefore, we option $C l^{*}\left(I_{\tau}(\mu, r), r\right) \leq \mu$.

Remark 2.5. Let $(X, \tau, \mathbf{I})$ be a fits. For each $\mu \in I^{X}$, we have $I_{\tau}\left(C l^{*}(\overline{1}-\mu, r), r\right) \neq \overline{1}-C l^{*}\left(I_{\tau}(\mu, r), r\right)$ as show by the following example.

Example 2.5. In Example 2.4, If we take $\mathbf{I}=\mathbf{I}^{0}$ for all $r \in I_{0}$, and let $\mu=\overline{0.7}$, then $\mu$ satisfies the above properties.

Corollary 2.4. Let $(X, \tau, \mathbf{I})$ be a fuzzy ideal topological space, such that $I_{\tau}\left(C l^{*}(\overline{1}-\mu, r), r\right) \neq \overline{1}-$ $C l^{*}\left(I_{\tau}(\mu, r), r\right)$. Then $\mu$ is r-FPIC iff $C l^{*}\left(I_{\tau}(\mu, r), r\right) \leq$ $\mu$. for each $\mu \in I^{X}$ and $r \in I_{0}$.

Theorem 2.5. Let $(X, \tau, \mathbf{I})$ be a fuzzy ideal topological space. For each $\lambda \in I^{X}$, we define an operator $\mathbf{I} C_{\tau}: I^{X} \rightarrow I$ as follows:

$\mathbf{I} C_{\tau}(\lambda, r)=\bigwedge\left\{\mu \in I^{X}: \quad \lambda \leq \mu, \quad \mu\right.$ is r-FIC $\}$.

For each $\lambda, \mu \in I^{X}$, the following properties are holds:

(1) $\mathbf{I} C_{\tau}(\overline{0}, r)=\overline{0}$.

(2) $\lambda \leq \mathbf{I} C_{\tau}(\lambda, r)$.

(3) $\mathbf{I} C_{\tau}(\lambda, r) \vee \mathbf{I} C_{\tau}(\mu, r) \leq \mathbf{I} C_{\tau}(\lambda \vee \mu, r)$.

(4) $\mathbf{I} C_{\tau}\left(\mathbf{I} C_{\tau}(\lambda, r), r\right)=\mathbf{I} C_{\tau}(\lambda, r)$.

(5) If $\lambda$ is r-FIC, iff $\lambda=\mathbf{I} C_{\tau}(\lambda, r)$.

(6) If $C_{\tau}(\lambda, r)$ is r-FIC, then $C_{\tau}\left(\mathbf{I} C_{\tau}(\lambda, r), r\right)=$ $\mathbf{I} C_{\tau}\left(C_{\tau}(\lambda, r), r\right)=C_{\tau}(\lambda, r)$.

Proof. (1), (2) and (5) are easily proved from the definition of $\mathbf{I} C_{\tau}$ and Lemma 1.1.

(3) Since $\lambda, \mu \leq \lambda \vee \mu$, we have

$$
\mathbf{I} C_{\tau}(\lambda, r) \vee \mathbf{I} C_{\tau}(\mu, r) \leq \mathbf{I} C_{\tau}(\lambda \vee \mu, r)
$$

(4) From (2) we have $\mathbf{I} C_{\tau}(\lambda, r) \leq \mathbf{I} C_{\tau}\left(\mathbf{I} C_{\tau}(\lambda, r), r\right)$. Now we show that $\mathbf{I} C_{\tau}(\lambda, r) \geq \mathbf{I} C_{\tau}\left(\mathbf{I} C_{\tau}(\lambda, r), r\right)$. Suppose that

$$
\mathbf{I} C_{\tau}(\lambda, r) \nsupseteq \mathbf{I} C_{\tau}\left(\mathbf{I} C_{\tau}(\lambda, r), r\right) .
$$

There exist $x \in X$ and $t \in(0,1)$ such that

$$
\mathbf{I} C_{\tau}(\lambda, r)(x)<t<\mathbf{I} C_{\tau}\left(\mathbf{I} C_{\tau}(\lambda, r), r\right)(x) .
$$

Since $\mathbf{I} C_{\tau}(\lambda, r)(x)<t$, by the definition $\mathbf{I} C_{\tau}$, there exists r-FIC, $\lambda_{1}$ with $\lambda \leq \lambda_{1}$ such that

$$
\mathbf{I} C_{\tau}(\lambda, r)(x) \leq \lambda_{1}(x)<t .
$$

Since $\lambda \leq \lambda_{1}$, we have $\mathbf{I} C_{\tau}(\lambda, r) \leq \lambda_{1}$. Again, by the definition $\mathbf{I} C_{\tau}$, we have $\mathbf{I} C_{\tau}\left(\mathbf{I} C_{\tau}(\lambda, r), r\right) \leq \lambda_{1}$. Hence $\mathbf{I} C_{\tau}\left(\mathbf{I} C_{\tau}(\lambda, r), r\right)(x) \leq \lambda_{1}(x)<t$. It is a contradiction for $(\mathbf{B})$. Thus

$$
\mathbf{I} C_{\tau}(\lambda, r) \geq \mathbf{I} C_{\tau}\left(\mathbf{I} C_{\tau}(\lambda, r), r\right) .
$$

(6) From (2) and $C_{\tau}(\lambda, r)$ is a r-FIC we have $\mathbf{I} C_{\tau}\left(C_{\tau}(\lambda, r), r\right)=C_{\tau}(\lambda, r)$.

we only show that

$$
C_{\tau}\left(\mathbf{I} C_{\tau}(\lambda, r), r\right)=C_{\tau}(\lambda, r) .
$$

Since $\lambda \leq \mathbf{I} C_{\tau}(\lambda, r)$

$$
C_{\tau}\left(\mathbf{I} C_{\tau}(\lambda, r), r\right) \geq C_{\tau}(\lambda, r) .
$$


Suppose that

$$
C_{\tau}\left(\mathbf{I} C_{\tau}(\lambda, r), r\right) C_{\tau}(\lambda, r) .
$$

There exist $x \in X$ and $r \in I_{0}$ such that

$$
C_{\tau}\left(\mathbf{I} C_{\tau}(\lambda, r), r\right)(x)>C_{\tau}(\lambda, r)(x)
$$

By the definition $C_{\tau}$, there exists $\nu \in I^{X}$, with $\lambda \leq \nu$ and $\tau(\overline{1}-\nu) \geq r$ such that

$$
C_{\tau}\left(\mathbf{I} C_{\tau}(\lambda, r), r\right)(x)>\nu(x) \geq C_{\tau}(\lambda, r)(x) .
$$

On the other hand, since $\nu=C_{\tau}(\nu, r), \lambda \leq \nu$, then

$\mathbf{I} C_{\mathbf{I}}(\lambda, r) \leq \mathbf{I} C_{\tau}(\nu, r)=\mathbf{I} C_{\tau}\left(C_{\tau}(\nu, r), r\right)=C_{\tau}(\nu, r)=\nu$.

Thus $C_{\tau}\left(\mathbf{I} C_{\tau}(\lambda, r), r\right) \leq \nu$.

It is a contradiction. Hence $C_{\tau}\left(\mathbf{I} C_{\tau}(\lambda, r), r\right) \leq C_{\tau}(\lambda, r)$.

Theorem 2.6. Let $(X, \tau, \mathbf{I})$ be a fits. For each $\lambda \in I^{X}$, we define an operator $\mathbf{I} I_{\tau}: I^{X} \rightarrow I$ as follows:

$\mathbf{I} I_{\tau}(\lambda, r)=\bigvee\left\{\mu \in I^{X}: \mu \leq \lambda, \quad \mu\right.$ is $\left.r-\mathbf{F I O}\right\}$.

Foreach $\mu \in I^{X}$, it holds the following properties:

(1) $\mathbf{I} I_{\tau}(\overline{1}-\mu, r)=\overline{1}-\left(\mathbf{I} C_{\tau}(\mu, r)\right)$.

(2) $\mathbf{I} I_{\tau}(\mu, r) \leq \mu \leq \mathbf{I} C_{\tau}(\mu, r)$.

(3) If $\mu$ is r-FIO iff $\mathbf{I} I_{\tau}(\mu, r)=\mu$.

Proof. (1) It is easily proved form the following:

$$
\begin{aligned}
& \overline{1}-\left(\mathbf{I} C_{\tau}(\lambda, r)\right) \\
& =\overline{1}-\bigwedge\left\{\mu \in I^{X}: \lambda \leq \mu, \mu \text { is } r-\text { FIC }\right\} \\
& =\bigvee\left\{\mu \in I^{X}: \overline{1}-\lambda \geq \overline{1}-\mu, \overline{1}-\mu \text { is } r-\text { FIO }\right\} \\
& =\mathbf{I} I_{\tau}(\overline{1}-\lambda, r) .
\end{aligned}
$$

(2) and (3) are easily proved form the definition of $\mathbf{I} I_{\tau}$ and Lemma 1.1.

\section{Decompositions of fuzzy continuity and fuzzy I-continuity}

Definition 3.1. A mapping $f:(X, \tau, \mathbf{I}) \rightarrow(Y, \eta)$ is called fuzzy I-continuous (resp. fuzzy pre-I-continuous, fuzzy $*$-I-continuous, fuzzy B-I-continuous, fuzzy semiI-continuous, fuzzy $\alpha$-I-continuous) if $f^{-1}(\mu)$ is r-FIO (resp. r-FPIO, r-fuzzy $*$-denes-in-itself, r-fuzzy B-I-set, r-FSIO, $\mathrm{r}-\mathbf{F} \alpha \mathbf{I O})$ for each $\eta(\mu) \geq r$ and $r \in I_{0}$.

According to Lemma 2.1-3 we have the following decomposition of fuzzy continuity and decomposition of fuzzy I-continuity.

Theorem 3.1. (1) A mapping $f:(X, \tau, \mathbf{I}) \rightarrow(Y, \eta)$ is called fuzzy continuous if and only if it is both fuzzy preI-continuous and fuzzy B-I-continuous.

(2) A mapping $f:(X, \tau, \mathbf{I}) \rightarrow(Y, \eta)$ is called fuzzy I-continuous if and only if it is both fuzzy pre-I-continuous and fuzzy $*$-I-continuous.
(3) A mapping $f:(X, \tau, \mathbf{I}) \rightarrow(Y, \eta)$ is called fuzzy $\alpha$ I-continuous if and only if it is both fuzzy pre-I-continuous and fuzzy semi-I-continuous.

Theorem 3.2. Let $f:(X, \tau, \mathbf{I}) \rightarrow(Y, \eta)$ be a function, then following statements are equivalent.

(1) A map $f$ is fuzzy $\alpha$-I-continuous.

(2) The inverse image of each r-fuzzy closed set in Y is r-F $\alpha \mathbf{I O}$.

(3) $C_{\tau}\left(I n t_{\tau}^{*}\left(C_{\tau}\left(f^{-1}(\lambda), r\right), r\right), r\right) \leq f^{-1}\left(C_{\eta}(\lambda), r\right)$, for each $\lambda \in I^{Y}$ and $r \in I_{0}$.

(4) $f\left(C_{\tau}\left(\operatorname{Int}_{\tau}^{*}\left(C_{\tau}(\mu, r), r\right), r\right)\right) \leq C_{\eta}(f(\mu), r)$, for each $\mu \in I^{X}$ and $r \in I_{0}$.

Proof. $\quad(1) \Leftrightarrow(2)$ : It easily proved form Definition 3.1, and $f^{-1}(\overline{1}-\mu)=\overline{1}-f^{-1}(\mu)$.

(2) $\Leftrightarrow(3)$ : For each $\lambda \in I^{Y}$ and $r \in I_{0}$. Since $C_{\eta}(\lambda, r)$ is r-fuzzy closed set in Y, by (2) $f^{-1}\left(C_{\eta}(\lambda, r)\right.$ is r-F $\alpha \mathbf{I C}$ and $\overline{1}-f^{-1}\left(C_{\eta}(\lambda, r)\right.$ is $\mathbf{r}-\mathbf{F} \alpha \mathbf{I O}$. Therefore,

$$
\begin{aligned}
\overline{1}- & f^{-1}\left(C_{\eta}(\lambda, r)\right. \\
& \leq I_{\tau}\left(C l^{*}\left(I_{\tau}\left(\overline{1}-f^{-1}\left(C_{\tau}(\lambda, r)\right), r\right), r\right)\right. \\
& =\overline{1}-C_{\tau}\left(\operatorname{Int}_{\tau}^{*}\left(C_{\tau}\left(f^{-1}\left(C_{\tau}(\lambda, r)\right), r\right), r\right), r\right) .
\end{aligned}
$$

Hence, we obtain

$$
f^{-1}\left(C_{\eta}(\lambda, r) \geq C_{\tau}\left(\operatorname{Int}_{\tau}^{*}\left(C_{\tau}\left(f^{-1}(\lambda), r\right), r\right), r\right) .\right.
$$

(3) $\Leftrightarrow$ (4): For each $\mu \in I^{X}$ and $r \in I_{0}$. By (3), we have

$$
\begin{aligned}
C_{\tau}\left(\operatorname{Int}_{\tau}^{*}\left(C_{\tau}(\mu, r), r\right)\right. & \leq C_{\tau}\left(\operatorname{Int}_{\tau}^{*}\left(C_{\tau}\left(f^{-1} f(\mu), r\right), r\right)\right. \\
& \leq f^{-1}\left(C_{\eta}(f(\mu), r)\right),
\end{aligned}
$$

and hence

$$
f\left(C_{\tau}\left(\operatorname{Int}_{\tau}^{*}\left(C_{\tau}(\mu, r), r\right), r\right)\right) \leq C_{\eta}(f(\mu), r) .
$$

(4) $\Leftrightarrow(1)$ : Let $\eta(\nu) \geq r$. Then by (4),

$$
\begin{aligned}
f\left(C _ { \tau } \left(\operatorname { I n t } _ { \tau } ^ { * } \left(C_{\tau}\right.\right.\right. & \left.\left.\left.\left(f^{-1}(\overline{1}-\nu)\right), r\right), r\right), r\right) \\
& \leq C_{\eta}\left(f f^{-1}(\overline{1}-\nu), r\right) \\
& \left.\leq C_{\eta}(\overline{1}-\nu), r\right)=\overline{1}-\nu .
\end{aligned}
$$

Thus,

$$
\begin{aligned}
C_{\tau}\left(\operatorname{Int}_{\tau}^{*}\left(C_{\tau}\left(f^{-1}(\overline{1}-\nu), r\right), r\right), r\right) & \leq f^{-1}(\overline{1}-\nu) \\
& \leq \overline{1}-f^{-1}(\nu) .
\end{aligned}
$$

Consequently, we have

$$
f^{-1}(\nu) \leq I_{\tau}\left(C l^{*}\left(I_{\tau}\left(f^{-1}(\nu), r\right), r\right), r\right) .
$$

This show that $f^{-1}(\nu)$ is r-F $\alpha \mathbf{I O}$. Thus, $f$ is fuzzy $\alpha$-Icontinuous.

Theorem 3.3. Let $f:(X, \tau, \mathbf{I}) \rightarrow(Y, \eta)$ be fuzzy $\alpha$-Icontinuous, then

(1) $f\left(C l^{*}(\mu, r)\right) \leq C_{\eta}(f(\mu), r)$, for each $\mu \in I^{X}$ is r-FPIO.

(2) $C l^{*}\left(f^{-1}(\lambda), r\right) \leq f^{-1}\left(C_{\tau}(\lambda, r)\right)$, for each $\lambda \in I^{Y}$ is r-FPIO. 
Proof. (1) If $\mu \in I^{X}$ is r-FPIO, then $\mu \leq I_{\tau}\left(C l^{*}(\mu, r), r\right)$. Thus, by Theorem 3.2 we have

$$
\begin{aligned}
f\left(C l^{*}(\mu, r)\right) & \leq f\left(C_{\tau}(\mu, r)\right) \\
& \leq f\left(C_{\tau}\left(I_{\tau}\left(C l^{*}(\mu, r), r\right), r\right)\right) \\
& \leq f\left(C_{\tau}\left(\operatorname{Int}_{\tau}^{*}\left(C_{\tau}(\mu, r), r\right), r\right)\right) \\
& \leq C_{\tau}(f(\mu), r) .
\end{aligned}
$$

(2) If $\lambda \in I^{Y}$ is r-FPIO, then $\lambda \leq I_{\tau}\left(C l^{*}(\lambda, r), r\right)$. Therefore, by Theorem 3.2, we have

$$
\begin{aligned}
& C l^{*}\left(f^{-1}(\lambda), r\right) \leq C_{\tau}\left(f^{-1}(\lambda), r\right) \\
& \quad \leq C_{\tau}\left(f^{-1}\left(I_{\tau}\left(C l^{*}(\lambda, r), r\right), r\right)\right) \\
& \quad \leq C_{\tau}\left(I_{\tau}\left(C l^{*}\left(I_{\tau}\left(f^{-1}\left(I_{\tau}\left(C l^{*}(\lambda, r), r\right)\right), r\right), r\right), r\right), r\right) \\
& \leq C_{\tau}\left(\operatorname{Int}_{\tau}^{*}\left(C_{\tau}\left(f^{-1}\left(I_{\tau}\left(C l^{*}(\lambda, r), r\right)\right), r\right), r\right), r\right) \\
& \leq f^{-1}\left(C_{\tau}\left(I_{\tau}\left(C l^{*}(\lambda, r), r\right), r\right)\right) \\
& \quad \leq f^{-1}\left(C_{\tau}(\lambda, r)\right) .
\end{aligned}
$$

Definition 3.2. A mapping $f:(X, \tau) \rightarrow(Y, \eta, \mathbf{I})$ is called fuzzy $\alpha$-I-open (resp. fuzzy semi-I-open, fuzzy pre-I-open, fuzzy $\beta$-I-open) if image of each $\mu \in I^{X}$ with $\tau(\mu) \geq r$ is r-F $\alpha$ IO (resp. r-FSIO, r-FPIO, r-F $\beta$ IO) set of Y.

Remark 3.1. By Definition 2.2, and Remark 2.1 we obtain the following diagram:

$$
\begin{array}{cc}
\text { fuzzy open } \Rightarrow \text { fuzzy } \alpha-\text { I-open } & \Rightarrow \text { fuzzy pre-I-open } \\
\Downarrow & \Downarrow \\
\text { fuzzy semi-I-open } & \Rightarrow \text { fuzzy } \beta \text {-I-open }
\end{array}
$$

Theorem 3.4. A mapping $f:(X, \tau) \rightarrow(Y, \eta, \mathbf{I})$ is called fuzzy $\alpha$-I-open if and only if it is fuzzy semi-I-open and fuzzy pre-I-open.

Proof. Form Lemma 2.1, the proof straightforward.

Theorem 3.5. A mapping $f:(X, \tau) \rightarrow(Y, \eta, \mathbf{I})$ is fuzzy $\alpha$-I-open if and only if for each $\mu \in I^{Y}$ and each $\tau(\overline{1}-\lambda) \geq r$, containing $f^{-1}(\mu)$, there exists $\nu \in I^{Y}$ r-F $\alpha$ IC containing $\mu$ such that $f^{-1}(\nu) \leq \lambda$.

Proof. Necessity. Let $\nu=\overline{1}-f(\overline{1}-\lambda)$. Since $f^{-1}(\mu) \leq \lambda$, we have $f(\overline{1}-\lambda) \leq \overline{1}-\mu$. Since $f$ is fuzzy $\alpha$-I-open, then $\nu$ is $\mathbf{r}-\mathbf{F} \alpha \mathbf{I C}$ and

$$
f^{-1}(\nu)=\overline{1}-f^{-1}(f(\overline{1}-\lambda)) \leq \overline{1}-(\overline{1}-\lambda)=\lambda .
$$

Sufficiency. Obvious.

Corollary 3.1. Let $f:(X, \tau, \mathbf{I}) \rightarrow(Y, \eta)$ be fuzzy $\alpha$ I-open. For each $\mu \in I^{Y}$, then

(1) $f^{-1}\left(C_{\tau}\left(I_{\tau}^{*}\left(C_{\tau}(\mu, r), r\right), r\right)\right) \leq C_{\eta}\left(f^{-1}(\mu), r\right)$.

(2) $f^{-1}\left(C l^{*}(\lambda, r)\right) \leq C_{\tau}\left(f^{-1}(\lambda), r\right)$.

Proof. For each $\mu \in I^{Y}$, then $C_{\tau}\left(f^{-1}(\mu), r\right)$ is f-fuzzy closed. By Theorem 3.5, there exists $\nu \in I^{Y}$ r-F $\alpha \mathbf{I C}$ containing $\mu$ such that $f^{-1}(\nu) \leq C_{\tau}\left(f^{-1}(\mu), r\right)$. Since $\overline{1}-\nu$ is $\mathrm{r}-\mathbf{F} \alpha \mathbf{I O}, f^{-1}(\overline{1}-\nu) \leq f^{-1}\left(I_{\tau}\left(C l^{*}\left(I_{\tau}(\overline{1}-\nu, r), r\right), r\right)\right)$ and

$$
\begin{aligned}
\overline{1}-f^{-1}(\nu) & \leq f^{-1}\left(\overline{1}-C_{\tau}\left(\operatorname{Int}_{\tau}^{*}\left(C_{\tau}(\nu, r), r\right), r\right)\right) \\
& \leq \overline{1}-f^{-1}\left(C_{\tau}\left(\operatorname{Int}_{\tau}^{*}\left(C_{\tau}(\nu, r), r\right), r\right)\right) .
\end{aligned}
$$

Therefore,

$f^{-1}\left(C_{\tau}\left(I n t_{\tau}^{*}\left(C_{\tau}(\nu, r), r\right), r\right)\right) \leq f^{-1}(\nu) \leq C_{\eta}\left(f^{-1}(\mu), r\right)$.

Thus, $f^{-1}\left(C_{\tau}\left(I_{\tau}^{*}\left(C_{\tau}(\mu, r), r\right), r\right)\right) \leq C_{\eta}\left(f^{-1}(\mu), r\right)$.

(2) Similarly.

Theorem 3.6. Let $f:(X, \tau, \mathbf{I}) \rightarrow(Y, \eta)$ be a mapping. For each $r \in I_{0}$, then following statements are equivalent.

(1) A map $f$ is called fuzzy I-continuous function.

(2) $f^{-1}(\mu)$ is r-FIC, in $\mathrm{X}$ for each $\mu \in I^{X}, r \in I_{0}$, With $\eta(\overline{1}-\mu) \geq r$.

(3) $f\left(\mathbf{I} C_{\tau}(\lambda, r)\right) \leq C_{\eta}(f(\lambda), r)$, for each $\lambda \in I^{X}$.

(4) $\mathbf{I} C_{\tau}\left(f^{-1}(\mu, r)\right) \leq f^{-1}\left(C_{\eta}(\mu, r)\right)$, for $\mu \in I^{Y}$.

(5) $f^{-1}\left(I_{\eta}(\mu, r)\right) \leq \mathbf{I} I_{\tau}\left(f^{-1}(\mu, r)\right)$, for each $\mu \in I^{Y}$.

Proof. $(1) \Leftrightarrow(2)$ : It easily proved form Definition 1.6(2), and $f^{-1}(\overline{1}-\mu)=\overline{1}-f^{-1}(\mu)$.

(2) $\Rightarrow(3)$ : Suppose there exist $\lambda \in I^{X}$ and $r \in I_{0}$ such that

$$
f\left(\mathbf{I} C_{\tau}(\lambda, r)\right) \not \leq C_{\tau}(f(\lambda), r) .
$$

There exist $y \in Y$ and $t \in I_{0}$ such that

$$
f\left(\mathbf{I} C_{\tau}(\lambda, r)\right)(y)>t>C_{\eta}(f(\lambda), r)(y) .
$$

If $f^{-1}(\{y\})=\emptyset$, it is a contradiction because $f\left(\mathbf{I} C_{\tau}(\lambda, r)\right)(y) \neq 0$.

If $f^{-1}(\{y\}) \neq \emptyset$, there exists $x \in f^{-1}(\{y\})$ such that

$$
f\left(\mathbf{I} C_{\tau}(\lambda, r)\right)(y) \geq \mathbf{I} C_{\tau}(\lambda, r)(x)>t>C_{\eta}(f(\lambda), r)(f(x)) .
$$

Since $C_{\eta}(f(\lambda), r)(f(x)) \leq t$, there exists $\eta(\overline{1}-\mu) \geq r$ with $f(\lambda) \leq \mu$ such that

$$
C_{\eta}(f(\lambda), r)(f(x)) \leq \mu(f(x)) \leq t .
$$

Moreover, $f(\lambda) \leq \mu$ implies $\lambda \leq f^{-1}(\mu)$. Form (2), $f^{-1}(\mu)$ is r-FIC. Thus,

$\mathbf{I} C_{\tau}(\lambda, r)(x) \leq f^{-1}(\mu)(x)=\mu(f(x))<t$. It is a contradiction for (A).

(3) $\Rightarrow(4)$ : For all $\mu \in I^{Y}, r \in I_{0}$, put $\lambda=f^{-1}(\mu)$. Form (3), we have

$$
f\left(\mathbf{I} C_{\tau}\left(f^{-1}(\mu), r\right)\right) \leq C_{\eta}\left(f\left(f^{-1}(\mu)\right), r\right) \leq C_{\eta}(\mu, r) .
$$

It implies

$$
\begin{aligned}
\mathbf{I} C_{\tau}\left(f^{-1}(\mu), r\right) & \leq f^{-1}\left(f\left(C_{\eta}\left(f^{-1}(\mu)\right), r\right)\right) \\
& \leq f^{-1}\left(C_{\eta}(\mu, r)\right) .
\end{aligned}
$$


$(4) \Rightarrow(5)$ : It easily proved form Theorems $2.6(1)$ and Theorem 1.2(1).

$(5) \Rightarrow(1)$ : Let $\eta(\mu) \geq r$. Then we have by definition $I_{\tau}$, $\mu=I_{\eta}(\mu, r)$. By (5) we have

$$
f^{-1}(\mu) \leq \mathbf{I} I_{\tau}\left(f^{-1}(\mu), r\right) .
$$

On the other hand, by Theorem 2.6(2),

$$
f^{-1}(\mu) \geq \mathbf{I} I_{\tau}\left(f^{-1}(\mu), r\right) .
$$

Thus, $f^{-1}(\mu)=\mathbf{I} I_{\tau}\left(f^{-1}(\mu), r\right)$ that is $f^{-1}(\mu)$ is r-FIO.

Analogous theorems to Theorem 3.6 can be given for the types of continuity in Definition 3.1.

Definition 3.3. Let $f:(X, \tau, \mathbf{I}) \rightarrow(Y, \eta, \mathbf{I})$ be a mapping.

(1) $f$ is called fuzzy I-irresolute if $f^{-1}(\mu)$ is r-FIO set of $\mathrm{X}$ for each r-FIO $\mu \in I^{Y}$ and $r \in I_{0}$.

(2) $f$ is called fuzzy I-irresolute open (resp. fuzzy Iopen) if $f(\mu)$ is r-FIO set of Y for each r-FIO $\mu \in I^{X}$ (resp. $\tau(\mu) \geq r$ ).

(3) $f$ is called fuzzy I-irresolute closed (resp. fuzzy I-closed) if $f(\mu)$ is r-FIC set of Y for each r-FIC $\mu \in I^{X}$ (resp. $\tau(\overline{1}-\mu) \geq r$.

The Following theorem is similarly proved as Theorem 3.6.

Theorem 3.7. Let $f:(X, \tau, \mathbf{I}) \rightarrow(Y, \eta, \mathbf{I})$ be a mapping. Then following statements are equivalent.

(1) A map $f$ is fuzzy I-irresolute.

(2) For each r-FIC $\mu \in I^{Y}, f^{-1}(\mu)$ is r-FIC.

(3) $f\left(\mathbf{I} C_{\tau}(\lambda, r)\right) \leq \mathbf{I} C_{\eta}(f(\lambda), r)$, for each $\lambda \in I^{X}$ and $r \in I_{0}$.

(4) $\mathbf{I} C_{\tau}\left(f^{-1}(\mu, r)\right) \leq f^{-1}\left(\mathbf{I} C_{\eta}(\mu, r)\right)$, for each $\mu \in$ $I^{Y}$ and $r \in I_{0}$.

(5) $f^{-1}\left(\mathbf{I} I_{\eta}(\mu, r)\right) \leq \mathbf{I} I_{\tau}\left(f^{-1}(\mu, r)\right)$, for each $\mu \in I^{Y}$ and $r \in I_{0}$.

Theorem 3.8. Let $f:(X, \tau, \mathbf{I}) \rightarrow(Y, \eta, \mathbf{I})$ be a bijective mapping. The following statements are equivalent.

(1) A map $f$ is fuzzy I-irresolute.

(2) $\left.\mathbf{I} I_{\eta}(f(\mu), r)\right) \leq f\left(\mathbf{I} I_{\tau}(\mu), r\right)$, for each $\mu \in I^{X}$.

Proof. $(1) \Rightarrow(2)$ : Let $f$ be fuzzy I-irresolute mapping and $\mu \in I^{X}$. Then $f^{-1}\left(\mathbf{I} I_{\eta}(f(\mu), r)\right)$ is r-FIO. Form Theorem 3.7(5), and the fact that $f$ is one-to-one we have

$f^{-1}\left(\mathbf{I} I_{\eta}(f(\mu), r)\right) \leq \mathbf{I} I_{\tau}\left(f^{-1}(f(\mu)), r\right)=\mathbf{I} I_{\tau}(\mu, r)$.

Again since $f$ is onto we have

$\mathbf{I} I_{\eta}(f(\mu), r)=f f^{-1}\left(\mathbf{I} I_{\eta}(f(\mu), r)\right) \leq f\left(\mathbf{I} I_{\tau}(\mu, r)\right)$.

$(2) \Rightarrow(1)$ : Let $\mu$ is r-FIO set of Y. Form Theorem 2.6(3), $\mu=\mathbf{I} I_{\eta}(\mu, r)$. By (2) we have

$f\left(\mathbf{I}_{\tau}\left(f^{-1}(\mu), r\right)\right) \geq \mathbf{I} I_{\eta}\left(f f^{-1}(\mu), r\right)=\mathbf{I} I_{\eta}(\mu, r)=\mu$ and

$\mathbf{I} I_{\tau}\left(f^{-1}(\mu), r\right)=f^{-1} f\left(\mathbf{I} I_{\tau}\left(f^{-1}(\mu), r\right)\right) \leq f^{-1}(\mu)$.

Thus, $f^{-1}(\mu)=\mathbf{I} I_{\tau}\left(f^{-1}(\mu), r\right)$. Thus, $f$ is fuzzy Iirresolute.

Theorem 3.9. Let $f:(X, \tau, \mathbf{I}) \rightarrow(Y, \eta, \mathbf{J})$ be fuzzy ideal topological space $f: X \rightarrow Y$ be a mapping. Then following statements are equivalent.

(1) $f$ is fuzzy I-irresolute open.

(2) $f\left(\mathbf{I} I_{\tau}(\lambda, r)\right) \leq \mathbf{I} I_{\eta}(f(\lambda), r)$, for each $\lambda \in I^{X}$ and $r \in I_{0}$.

(3) $\mathbf{I} I_{\tau}\left(f^{-1}(\mu), r\right) \leq f^{-1}\left(\mathbf{I} I_{\eta}(\mu, r)\right)$, for each $\mu \in I^{Y}$ and $r \in I_{0}$.

(4) For any $\mu \in I^{Y}$ and any r-FIC $\lambda \in I^{X}$ with $f^{-1}(\mu) \leq \lambda$, there exists a r-FIC $\rho \in I^{Y}$ with $\mu \leq \rho$ such that $f^{-1}(\rho) \leq \lambda$.

\section{Proof.}

$(1) \Rightarrow(2)$ : For each $\lambda \in I^{X}$. Since $\mathbf{I} I_{\tau}(f(\lambda), r) \leq$ $\lambda$ form Theorem 2.6(2), we have $f\left(\mathbf{I} I_{\tau}(\lambda, r)\right) \leq f(\lambda)$. form (1), $f\left(\mathbf{I} I_{\tau}(\lambda, r)\right)$ is r-FIO. Therefore $f\left(\mathbf{I} I_{\tau}(\lambda, r)\right) \leq$ $\mathbf{I} I_{\eta}(f(\lambda), r)$.

$(2) \Rightarrow(3)$ : For all $\mu \in I^{Y}$ and $r \in I_{0}$, put $\lambda=f^{-1}(\mu)$ form (2). Then

$f\left(\mathbf{I} I_{\tau}\left(f^{-1}(\mu), r\right)\right) \leq \mathbf{I} I_{\eta}\left(f\left(f^{-1}(\mu)\right), r\right) \leq \mathbf{I} I_{\eta}(\mu, r)$.

It implies $\mathbf{I} I_{\tau}\left(f^{-1}(\mu), r\right) \leq f^{-1}\left(\mathbf{I} I_{\eta}(\mu, r)\right)$.

$(3) \Rightarrow(4)$ : Let $\lambda$ be r-FIC set of X such that $f^{-1}(\mu) \leq$ $\lambda$. Since $\overline{1}-\lambda \leq f^{-1}(\overline{1}-\mu)$ and $\mathbf{I} I_{\tau}(\overline{1}-\lambda, r)=\overline{1}-\lambda$,

$$
\mathbf{I} I_{\tau}(\overline{1}-\lambda, r)=\overline{1}-\lambda \leq \mathbf{I} I_{\tau}\left(f^{-1}(\overline{1}-\mu), r\right) .
$$

From (3),

$$
\overline{1}-\lambda \leq \mathbf{I} I_{\tau}\left(f^{-1}(\overline{1}-\mu), r\right) \leq f^{-1}\left(\mathbf{I} I_{\eta}(\overline{1}-\mu, r)\right) .
$$

It implies

$$
\begin{aligned}
\lambda & \geq \overline{1}-f^{-1}\left(\mathbf{I} I_{\eta}(\overline{1}-\mu), r\right) \\
& =f^{-1}\left(\overline{1}-\mathbf{I} I_{\eta}(\overline{1}-\mu, r)\right) \\
& =f^{-1}\left(\mathbf{I} C_{\eta}(\mu, r)\right) .
\end{aligned}
$$

Hence there exists a r-FIC $\mathbf{I} C_{\eta}(\mu, r)$ with $\mu \leq \mathbf{I} C_{\eta}(\mu, r)$ such that $f^{-1}\left(\mathbf{I} C_{\eta}(\mu, r)\right) \leq \lambda$.

(4) $\Rightarrow$ (1) Let $\omega$ be r-FIO of X. Put $\mu=\overline{1}-f(\omega)$ and $\lambda=\overline{1}-\omega$ such that $\lambda$ is r-FIC. We obtain

$$
\begin{aligned}
f^{-1}(\mu) & =f^{-1}(\overline{1}-f(\omega)) \\
& =\overline{1}-f^{-1}(f(\omega)) \\
& \leq \overline{1}-\omega=\lambda .
\end{aligned}
$$


Form (4) there exists a r-FIC set $\rho$ with $\mu \leq \rho$ such that $f^{-1}(\rho) \leq \lambda=\overline{1}-\omega$. It implies $\omega \leq \overline{1}-f^{-1}(\rho)=$ $f^{-1}(\overline{1}-\rho)$. Thus, $f(\omega) \leq f\left(f^{-1}(\overline{1}-\rho)\right)=\overline{1}-\rho$. On the other hand, since $\mu \leq \rho$,

$$
f(\omega)=\overline{1}-\mu \geq \overline{1}-\rho .
$$

Hence $f(\omega)=\overline{1}-\rho$, that is, $f(\omega)$ is r-FIO.

Theorem 3.10 is similarly proved from Theorem 3.9.

Theorem 3.10. Let $(X, \tau, \mathbf{I})$ and $(Y, \eta, \mathbf{I})$ be fuzzy ideal topological spaces $f: X \rightarrow Y$ be a mapping. Then following statements are equivalent.

(1) $f$ is fuzzy I-irresolute closed.

(2) $f\left(\mathbf{I} C_{\tau}(\lambda, r)\right) \leq \mathbf{I} C_{\eta}(f(\lambda), r)$, for each $\lambda \in I^{X}$ and $r \in I_{0}$.

(3) For any $\mu \in I^{Y}$ and any r-FIO $\lambda \in I^{X}$ with $f^{-1}(\mu) \leq \lambda$, there exists a r-FIO $\rho \in I^{Y}$ with $\mu \leq \rho$ such that $f^{-1}(\rho) \leq \lambda$.

Theorem 3.11. Let $(X, \tau, \mathbf{I})$ and $(Y, \eta)$ be fuzzy ideal topological space. A mapping $f: X \rightarrow Y$ be a fuzzy I-open. Then the following statements are holed.

(1) $f\left(I_{\tau}(\lambda, r)\right) \leq \mathbf{I} I_{\eta}(f(\lambda), r)$, for each $\lambda \in I^{X}$ and $r \in I_{0}$.

(2) $I_{\tau}\left(f^{-1}(\mu), r\right) \leq f^{-1}\left(\mathbf{I} I_{\eta}(\mu, r)\right)$, for each $\mu \in I^{Y}$ and $r \in I_{0}$.

(3) For any $\mu \in I^{Y}$ and $\tau(\overline{1}-\lambda) \geq r$ such that $f^{-1}(\mu) \leq \lambda$, there exists a r-FIC set $\rho \in I^{Y}$

with $\mu \leq \rho$ such that $f^{-1}(\rho) \leq \lambda$.

Proof. (1) For each $\lambda \in I^{X}$ since $I_{\tau}(\lambda, r) \leq \lambda$, by Theorem 1.2(3). Then $f\left(I_{\tau}(\lambda, r)\right) \leq f(\lambda)$. From (1), $f\left(I_{\tau}(\lambda, r)\right)$ is r-FIO. Therefore

$$
f\left(I_{\tau}(\lambda, r)\right) \leq \mathbf{I} I_{\eta}(f(\lambda), r)
$$

(2) For all $\mu \in I^{Y}$ and $r \in I_{0}$, put $\lambda=f^{-1}(\mu)$ form (2). Then

$$
f\left(I_{\tau}\left(f^{-1}(\mu), r\right)\right) \leq \mathbf{I} I_{\eta}\left(f\left(f^{-1}(\mu)\right), r\right)=\mathbf{I} I_{\eta}(\mu, r) .
$$

It implies $I_{\tau}\left(f^{-1}(\mu), r\right) \leq f^{-1}\left(\mathbf{I} I_{\eta}(\mu, r)\right)$.

(3) Let $\tau(\overline{1}-\lambda) \geq r$ set of $X$ such that $f^{-1}(\mu) \leq \lambda$. Since $\underline{1}-\lambda \leq f^{-1}(\underline{1}-\mu)$ and $I_{\tau}(\underline{1}-\lambda, r)=\underline{1}-\lambda$.

$$
I_{\tau}(\underline{1}-\lambda, r)=\underline{1}-\lambda \leq I_{\tau}\left(f^{-1}(\underline{1}-\mu), r\right) .
$$

Form (2), we have It implies

$$
\underline{1}-\lambda \leq I_{\tau}\left(f^{-1}(\underline{1}-\mu), r\right) \leq f^{-1}\left(\mathbf{I} I_{\eta}(\underline{1}-\mu, r)\right) .
$$

$$
\begin{aligned}
\lambda & \geq \underline{1}-f^{-1}\left(\mathbf{I} I_{\eta}(\underline{1}-\mu), r\right) \\
& =f^{-1}\left(\underline{1}-\mathbf{I} I_{\eta}(\underline{1}-\mu, r)\right) \\
& =f^{-1}\left(\mathbf{I} C_{\eta}(\mu, r)\right) .
\end{aligned}
$$

Hence there exists a r-FIC $\mathbf{I} C_{\eta}(\mu, r) \in I^{Y}$ with $\mu \leq$ $\mathbf{I} C_{\eta}(\mu, r)$ such that $f^{-1}\left(\mathbf{I} C_{\eta}(\mu, r)\right) \leq \lambda$.

Theorem 3.12 is similarly proved from Theorem 3.11 .

Theorem 3.12. Let $(X, \tau)$ and $(Y, \eta, \mathbf{I})$ be fuzzy ideal topological spaces. A mapping $f: X \rightarrow Y$ be a fuzzy I-closed. Then following statements are holed.

(1) $f\left(C_{\tau}(\lambda, r)\right) \leq \mathbf{I} C_{\eta}(f(\lambda), r)$, for $\lambda \in I^{X,} r \in I_{0}$.

(2) For any $\lambda \in I^{Y}$ and $\tau(\mu) \geq r$ such that $f^{-1}(\lambda) \leq \mu$, there exists a r-FIO with $\lambda \leq \rho$ such that $f^{-1}(\rho) \leq \mu$.

Theorem 3.13. Let $(X, \tau, \mathbf{I})$ and $(Y, \eta, \mathbf{J})$ be fuzzy ideal topological space and $f: X \rightarrow Y$ be a bijective mapping

(1) $f$ is a fuzzy I-irresolute closed iff $f^{-1}\left(\mathbf{I} C_{\eta}(\mu, r)\right) \geq$ $\mathbf{I} C_{\tau}\left(f^{-1}(\mu), r\right)$, for each $\mu \in I^{Y}$.

(2) $f$ is a fuzzy I-irresolute closed iff fuzzy I-irresolute open for each $\mu \in I^{X}$ and $r \in I_{0}$.

Proof. $1(\Rightarrow)$ Let $f$ be a fuzzy I-irresolute closed. Form Theorem 3.10(2), for each $\mu \in I^{X}$ and $r \in I_{0}$.

$$
f\left(\mathbf{I} C_{\tau}(\lambda, r)\right) \leq \mathbf{I} C_{\eta}(f(\lambda), r) .
$$

For all $\mu \in I^{Y}$ and $r \in I_{0}$ put $\lambda=f^{-1}(\mu)$. Since $f$ is onto, $f f^{-1}(\mu)=\mu$. Thus

$$
\begin{aligned}
f\left(\mathbf{I} C_{\tau}\left(f^{-1}(\mu), r\right)\right) & \leq \mathbf{I} C_{\eta}\left(f\left(f^{-1}(\mu)\right), r\right) \\
& =\mathbf{I} C_{\eta}(\mu, r) .
\end{aligned}
$$

It implies

$$
\begin{aligned}
\mathbf{I} C_{\tau}\left(f^{-1}(\mu), r\right) & =f^{-1}\left(f\left(\mathbf{I} C_{\tau}\left(f^{-1}(\mu), r\right)\right)\right) \\
& \leq f^{-1}\left(\mathbf{I} C_{\eta}(\mu, r)\right) .
\end{aligned}
$$

$1(\Leftarrow)$ Put $\mu=f(\lambda)$. Since $f$ is injective

$$
f^{-1}\left(\mathbf{I} C_{\eta}(f(\lambda), r)\right) \leq \mathbf{I} C_{\tau}\left(f^{-1}(f(\lambda)), r\right)=\mathbf{I} C_{\tau}(\lambda, r)
$$

Since $f$ is onto $\mathbf{I} C_{\eta}(f(\lambda), r) \leq f\left(\mathbf{I} C_{\tau}(\lambda, r)\right)$.

(2) It easily proved from:

$$
\begin{aligned}
& f^{-1}\left(\mathbf{I} C_{\eta}(\mu, r)\right) \leq \mathbf{I} C_{\tau}\left(f^{-1}(\mu), r\right) \\
& \Leftrightarrow \overline{1}-f^{-1}\left(\mathbf{I} I_{\eta}(\overline{1}-\mu, r)\right) \leq \overline{1}-\mathbf{I} I_{\tau}\left(\overline{1}-f^{-1}(\mu), r\right) . \\
& \Leftrightarrow f^{-1}\left(\mathbf{I} I_{\eta}(\overline{1}-\mu, r)\right) \geq \mathbf{I} I_{\tau}\left(f^{-1}(\overline{1}-\mu), r\right) .
\end{aligned}
$$

Form above theorems we have the following theorem.

Theorem 3.14. Let $(X, \tau, \mathbf{I})$ and $(Y, \eta, \mathbf{I})$ be fuzzy ideal topological spaces and $f: X \rightarrow Y$ be mappings. Then following statements are equivalent.

(1) $f$ is fuzzy I-irresolute and fuzzy I-irresolute open.

(2) $f$ is fuzzy I-irresolute and fuzzy I-irresolute closed.

(3) $f\left(\mathbf{I} I_{\tau}(\lambda, r)\right) \leq \mathbf{I} I_{\eta}(f(\lambda), r)$, for each $\lambda \in I^{X}$ and $r \in I_{0}$. 
(4) $f\left(\mathbf{I} C_{\tau}(\lambda, r)\right) \leq \mathbf{I} C_{\eta}(f(\lambda), r)$, for each $\lambda \in I^{X}$, $r \in I_{0}$.

(5) $\mathbf{I} I_{\tau}\left(f^{-1}(\mu), r\right) \leq f^{-1}\left(\mathbf{I} I_{\eta}(\mu, r)\right)$, for each $\mu \in I^{Y}$ and $r \in I_{0}$.

(6) $\mathbf{I} C_{\tau}\left(f^{-1}(\mu), r\right) \leq f^{-1}\left(\mathbf{I} C_{\eta}(\mu, r)\right)$, for each $\mu \in I^{Y}$ and $r \in I_{0}$.

Theorem 3.15. Let $f:(X, \tau, \mathbf{I}) \rightarrow(Y, \eta, \mathbf{I})$ and $g:(Y, \eta, \mathbf{I}) \rightarrow(Z, \gamma)$ be a mapping. the following statements are hold.

(1) If $f$ and $g$ is fuzzy I-irresolute, then $g \circ f$ is fuzzy I-irresolute.

(2) If $f$ is fuzzy I-irresolute and $g$ is fuzzy I-continuous, then $g \circ f$ is is fuzzy I-continuous.

(3) If $f$ and $g$ is fuzzy I-irresolute open, then $g \circ f$ is fuzzy I-irresolute open.

Proof. Obvious.

\section{References}

[1] Chang C.L. "Fuzzy topological spaces." J. Math. Anal. Appl. vol. 24, pp. 182-190, 1968.

[2] Chattopadhyay K.C, Hazra R.N, Samanta S.K. "Gradation of openness: fuzzy topology," Fuzzy Sets and Systems, vol. 49, pp. 237-42, 1992.

[3] Chattopadhyay K.C, Samanta S.K. "Fuzzy topology: fuzzy closure operator, fuzzy compactness and fuzzy connectedness," Fuzzy Sets and Systems, vol. 54, pp. 207-12, 1993.

[4] El-baki S.A, Zahran A.M, Abbas S.E, Saber Y.M. "On Fuzzy ideal topological spaces," to appear in Applied Mathematical Sciences, 2008.

[5] El Gayyar M.K, Kerre E.E. Ramadan A.A. “Almost compactness and near compactness in smooth topological spaces," Fuzzy Sets and Systems, vol. 62, pp. 193-202, 1994.

[6] EL Naschie M.S, Rossler Oed G. "Information and diffusion in quantum physics." Chaos, Solitons \& Fractals, vol. 7, no.5, [special issue] 1996.

[7] El Naschie M.S. "On the uncertainty of Cantorian geometry and the two-slit experiment." Chaos, Solitons \& Fractals, vol. 9, pp. 517-29, 1998

[8] El Naschie M.S. "On the unification of heterotic strings, M theory and $\varepsilon^{(\infty)}$ theory." Chaos, Solitons \& Fractals, vol. 11, pp. 2397-2408, 2000.

[9] El Naschie M.S. "A review of E-infinity theory and the mass spectrum of high energy particle physics." Chaos, Solitons \& Fractals, vol. 19, pp. 209-236, 2004.
[10] El Naschie M.S. "Quantum gravity from descriptive set theory." Chaos, Solitons \& Fractals, vol. 19, pp. 1339-1344, 2004.

[11] El Naschie M.S. "Quantum gravity, Clifford algebras, fuzzy set theory and the fundamental constants of nature." Chaos, Solitons \& Fractals, vol. 20, pp. 437-450, 2004.

[12] El Naschie M.S. "The simplistic vacuum, exotic quasiparticles and gravitational instanton." Chaos, Solitons \& Fractals, vol. 22, pp. 1-11, 2004.

[13] El Naschie M.S. "On a fuzzy Kahler-like manifold which is consistent with the two slit experiment." Int $J$ Nonlinear Sci Numer Simulat, vol. 6, pp. 95-98, 2005.

[14] El Naschie M.S. "Topics in the mathematical physics of E-infinity theory." Chaos, Solitons \& Fractals, vol. 30, pp. 656-663, 2006.

[15] El Naschie M.S. "Elementary prerequisite for E-infinity (recommended background readings in nonlinear dynamics, geometry and topology)." Chaos, Solitons \& Fractals, vol. 30, no.3, pp. 579-605, 2006.

[16] El Naschie M.S. "Advanced prerequisite for E-infinity theory." Chaos, Solitons \& Fractals, vol. 30, pp. 636-641, 2006.

[17] Hatir H, Jafari S. "Fuzzy semi-I-open and Fuzzy semi-I-continuity via fuzzy idealization," Chaos, Solitons \& Fractals, vol. 34, no.4, pp. 1220-1224, 2007.

[18] Hutton B, Reilly I. "Separation axioms in fuzzy topological spaces," Fuzzy Sets and Systems, vol. 3, pp. 93-104, 1980.

[19] Kim Y.C. Ko J.M. "r-generalized fuzzy closed sets." J Fuzzy Math, vol. 12, no.1, pp. 7-21, 2004.

[20] Kim Y.C. "r-fuzzy semi-open sets in fuzzy bitopolgical space," Far East J. Math. Sic Spiecial, FJMS vol. 11, pp. 221-236, 2000.

[21] Lowen R. "Fuzzy topological spaces and fuzzy compactness," J. Math. Anal. Appl, vol. 56, pp. 621-633, 1976.

[22] Nasef A.A, Mahmoud R.A. "Some topological applications via fuzzy ideals." Chaos, Solitons \& Fractals, vol. 13, pp. 825-831, 2002.

[23] Ramadan A.A. "Smooth topological spaces," Fuzzy Sets and Systems, vol. 48, pp. 371-375, 1992.

[24] Ramadan A.A, Abbas S.E, Kim Y.C. "Fuzzy irresolute functions in smooth fuzzy topological space." J Fuzzy Math, vol.9, no.4, pp. 865-877, 2001.

[25] Ramadan A.A, Abbas S.E, Kim Y.C. "On weaker forms of continuity is Sostak's fuzzy topology," Indian J. Pure and Appl, vol. 34, no.2, pp. 311-333, 2003. 
[26] Ramadan A.A, Abde-Sattar M.A, El Gayyar M.K. Al-Azhar University, Assuit, Egypt Smooth L-ideal, Quaestiones Mathematicae 2000.

Research Area: Fuzzy topology, General topology

E-mail : zahran15@hotmail.com

[27] Sarkar D. "Fuzzy ideal theory, fuzzy local function and generated fuzzy topology, fuzzy topology." Fuzzy Sets and Systems, vol. 87, pp. 117-123, 2001.

[28] Sostak A.P. “On a fuzzy topological structure.” Suppl. Rend. Circ. Mat Palermo Ser II, vol. 11, pp. 89-103, 1985.

[29] Sostak A.P. "On some modifications of fuzzy topologies.” Mat Vesnik, vol. 41, pp. 51-64, 1989.

\author{
Ahmed M. Zahran \\ M.Sc: 1986 \\ Ph.D: 1990 \\ Assoc. Professor: 1996 \\ Professor: 2002 \\ Department of Mathematics, Faculty of Science (Assuit)
}

\section{S. Ahmed Abd El-Baki}

M.Sc: 1986

Ph.D: 1991

Department of Mathematics,

Faculty of Science

Assuit University, Assuit, Egypt

Research Area: Fuzzy topology

E-mail : mazab57@yahoo.com

\section{Yaser Mohammed Saber}

M.Sc. : 2006

Department of Mathematics, Faculty of Science (Assuit)

Al-Azhar University, Assuit, Egypt

Research Area: Fuzzy topology, General topology

E-mail : m.ah75@Yahoo.com 